\title{
A Reduced-PAR Opportunistic STBC Scheme for Frequency-Selective Channels in the Presence of Frequency Offset
}

\author{
Dandan Wang, Student Member, IEEE, Hlaing Minn, Senior Member, IEEE, \\ and Naofal Al-Dhahir, Fellow, IEEE
}

\begin{abstract}
Opportunistic space-time block codes (STBC) have been investigated for flat-fading channels in the literature. In this paper, we present a novel scheme for integrating them with orthogonal frequency division multiplexing (OFDM) for frequency-selective channels with an unequal error protection capability making it attractive for multimedia and multicasting applications. In addition, our new scheme optimizes the tradeoff between coding gain and peak-to-average ratio (PAR) while minimizing inter-carrier interference in the presence of carrier frequency offset. We show that our scheme achieves a $2 \mathrm{~dB}$ reduction in PAR over the conventional scheme.
\end{abstract}

Index Terms-STBC, OFDM, PAR, ICI.

\section{INTRODUCTION}

$\mathbf{S}$ PACE-TIME block coding (STBC) has received considerable attention in academic and industrial circles due to its advantages [1][2], including low-complex decoding at the receiver. Recently, diversity-embedding space-time codes (DEC) were proposed to enable unequal error protection by embedding a high-diversity code within a high-rate code [3][4][5]. More specifically, the information symbols are encoded into layers each at a prescribed rate diversity operating point. Variable diversity levels are provided to different information layers according to their QoS requirements which makes DEC especially attractive for multimedia and multicasting applications. Therefore, the high-rate code of DEC opportunistically takes advantage of good channel realizations while the embedded high-diversity code ensures that at least part of the information is decoded reliably. However, previous DEC constructions in [3][4][5] were presented for flatfading channels only. To utilize the frequency diversity gain of frequency-selective channels, STBCs have been combined with orthogonal frequency division multiplexing (OFDM) by implementing STBC at a block level instead of a symbol level, commonly known as STBC-OFDM [6]. A straightforward approach to combine DEC with OFDM is to use the general STBC-OFDM structure. However, this will result in a high peak to average ratio (PAR). Large PAR is a well-known problem to OFDM systems which require a larger backoff and hence power loss for nonlinear amplifiers [7][8]. In

Manuscript received February 16, 2007; revised May 3, 2007; accepted June 11,2007 . The associate editor coordinating the review of this paper and approving it for publication was L. Lampe. This work was supported in parts by the Erik Jonsson School Research Excellence Initiative, the University of Texas at Dallas, USA, the National Science Foundation (NSF) under Contracts CCF 04-30654 and DMS 05-28010. The material in this paper was presented in part in IEEE ICASSP, Hawaii, USA, April, 2007.

The authors are with the Department of Electrical Engineering, University of Texas at Dallas, Richardson, TX, 75083 (e-mail: \{dxw053000, hlaing.minn, aldhahir\}@utdallas.edu).

Digital Object Identifier 10.1109/TWC.2008.070200. addition, OFDM receivers are sensitive to carrier frequency offsets which result in inter-carrier interference (ICI) [9][10]. In the presence of multiple transmit antennas, the effects of carrier frequency offsets are even more pronounced [11]. Our proposed opportunistic STBC-OFDM scheme is designed to be robust against residual frequency offset while ensuring a favorable performance tradeoff between coding gain and PAR.

\section{PAR REDUCTION DESIGN FOR OpPORTUNistiC STBC-OFDM}

In this section, we describe our proposed opportunistic STBC-OFDM scheme. As a basic example, we consider the following DEC design for 2 transmit antennas [5]

$$
X_{a, b}=\left(\begin{array}{cc}
a(0) & \frac{b(0)}{K} \\
-\frac{b^{*}(1)}{K} & -a^{*}(0)
\end{array}\right),
$$

where $(.)^{*}$ denotes the complex conjugation. Information symbols are transmitted in 2 layers. Layer A consists of symbol $a(0)$ while Layer B consists of symbols $b(0)$ and $b(1)$ resulting in an overall rate of 1.5 symbols per channel use. The scalar $K(K>1)$ is designed to ensure a diversity level of 2 for Layer A and 1 for Layer B. An optimum design of $K$ will be given in Section IV. In this paper, we choose all the information symbols from the unit-energy QPSK constellation. Extensions to other constellations are straightforward. If we switch the two signals on the two time slots as

$$
X_{a, b}^{\prime}=\left(\begin{array}{cc}
\frac{b(0)}{K} & a(0) \\
-a^{*}(0) & -\frac{b^{*}(1)}{K}
\end{array}\right),
$$

then this switched DEC (S-DEC) maintains the same performance as DEC since it can be easily switched back at the receiver. Motivated by this observation, we propose our new opportunistic STBC-OFDM scheme (illustrated in Fig. 1), where the basic idea is to implement hybrid DEC/S-DEC at the OFDM symbol level. Let $J_{a}$ denote the set of sub-carriers on which DEC is implemented while $J_{b}$ denotes the set of the sub-carriers on which S-DEC is implemented. Suppose the size of $J_{a}$ is $m$ and let $N$ denote the overall number of sub-carriers. Then, the size of $J_{b}$ is $N-m$. In addition, let $a_{0, n}, b_{0, n}$ and $b_{1, n}$ denote the signals $a(0), b(0)$ and $b(1)$, respectively, associated with the $n$-th $(n=0,1, \ldots, N-1)$ sub-carrier.

Consider the first transmit antenna. From (1) and (2), we obtain the signal transmitted on the $n$th sub-carrier in the first time slot and second time slot, respectively, as

$$
\tilde{s}_{1, n}=\left\{\begin{array}{c}
a_{0, n}, n \in J_{a} \\
\frac{b_{0, n}}{K}, n \in J_{b}
\end{array} \text { and } \tilde{s}_{2, n}=\left\{\begin{array}{c}
\frac{b_{0, n}}{K}, n \in J_{a} \\
a_{0, n}, n \in J_{b}
\end{array}\right.\right.
$$




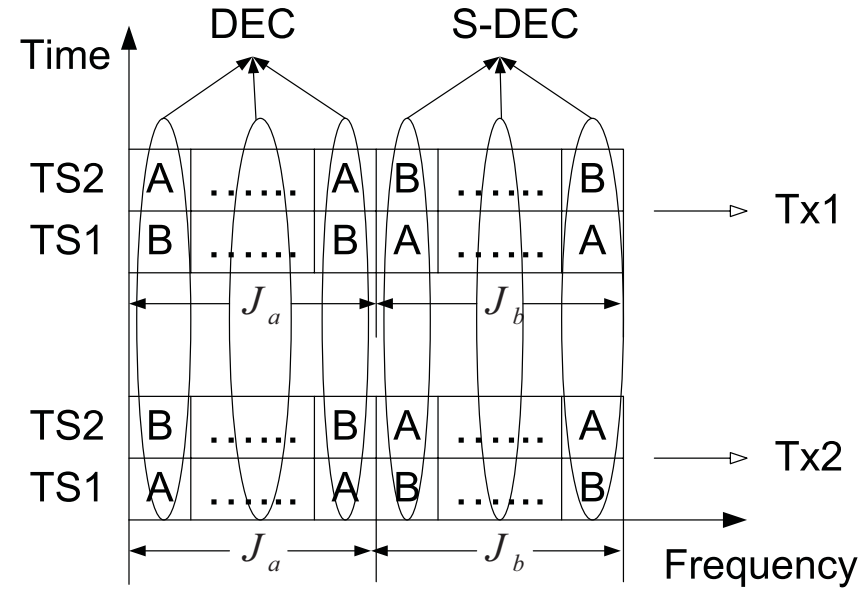

Fig. 1. Illustration of one STBC-OFDM block.

Let $\left\{s_{1, p}\right\}$ and $\left\{s_{2, p}\right\}$ denote the low-pass-equivalent discretetime signals obtained with an over-sampling factor of $M$ from the first and second OFDM symbols, respectively. Then, we obtain [12]

$$
\begin{aligned}
s_{1, p}= & \frac{1}{M N}\left(\sum_{n=0, n \in J_{a}}^{N-1} a_{0, n} e^{\frac{j 2 \pi n p}{M N}}+\sum_{n=0, n \in J_{b}}^{N-1} \frac{b_{0, n}}{K} e^{\frac{j 2 \pi n p}{M N}}\right), \\
& p=0,1, \ldots, M N-1,
\end{aligned}
$$

whose amplitudes are upper bounded as

$$
\begin{aligned}
\left|s_{1, p}\right| & \leq \frac{1}{M N}\left(\sum_{n=0, n \in J_{a}}^{N-1}\left|a_{0, n}\right|\left|e^{\frac{j 2 \pi n p}{M N}}\right|+\sum_{n=0, n \in J_{b}}^{N-1}\left|\frac{b_{0, n}}{K} \| e^{\frac{j 2 \pi n p}{M N}}\right|\right) \\
& =\frac{1}{M N}\left(\frac{N-m}{K}+m\right) .
\end{aligned}
$$

Similarly, we obtain

$$
\left|s_{2, p}\right| \leq \frac{1}{M N}\left(\frac{m}{K}+N-m\right) .
$$

Let $\mathbf{c}=\left\{s_{1,0}, s_{1,1}, \ldots, s_{1, M N-1}, s_{2,0}, s_{2,1}, \ldots, s_{2, M N-1}\right\}$ denote the signals transmitted on the first antenna during one STBC-OFDM block and $c(p)$ represent the $p$ th element of $\mathbf{c}$. By using a sufficiently large $M$, the PAR of the continuoustime STBC-OFDM block can be approximated as follows [12]

$$
\mathrm{PAR}=\frac{\max _{p}|c(p)|^{2}}{\frac{1}{2 M N} \sum_{p=0}^{2 M N-1}|c(p)|^{2}} .
$$

According to the Parseval's theorem, we have $\sum_{p=0}^{M N-1}\left|s_{1, p}\right|^{2}=\frac{\sum_{n=0}^{N-1}\left|\tilde{s}_{1, n}\right|^{2}}{M N}=\frac{\frac{N-m}{K^{2}}+m}{M N} \quad$ and $\sum_{p=0}^{M N-1}\left|s_{2, p}\right|^{2}=\frac{\sum_{n=0}^{N-1}\left|\tilde{s}_{2, n}\right|^{2}}{M N}=\frac{\frac{m}{K^{2}}+N-m}{M N}$. Hence, we obtain

$$
\begin{aligned}
& \frac{1}{2 M N} \sum_{p=0}^{2 M N-1}|c(p)|^{2}=\frac{\sum_{p=0}^{M N-1}\left|s_{1, p}\right|^{2}+\sum_{p=0}^{M N-1}\left|s_{2, p}\right|^{2}}{2 M N} \\
& =\frac{\frac{\frac{N-m}{K^{2}}+m+\frac{m}{K^{2}}+N-m}{N M}}{2 N M}=\frac{1+\frac{1}{K^{2}}}{2 N M^{2}} .
\end{aligned}
$$

The maximum PAR over all possible codewords is

$\mathrm{PAR}_{\max }=\max _{a_{0, n}, b_{0, n}, b_{1, n}}\{\mathrm{PAR}\}= \begin{cases}\frac{2\left(\frac{m}{K}+N-m\right)^{2}}{N\left(1+\frac{1}{K^{2}}\right)} & m \leq \frac{N}{2} \\ \frac{2\left(\frac{N-m}{K}+m\right)^{2}}{N\left(1+\frac{1}{K^{2}}\right)} & m \geq \frac{N}{2}\end{cases}$

The optimum $m$ is obtained by minimizing the maximum PAR as follows

$$
m_{\mathrm{opt}}=\underset{m}{\arg \min }\left\{\mathrm{PAR}_{\max }\right\}=\frac{N}{2},
$$

and the corresponding maximum PAR is

$$
\mathrm{PAR}_{\max }=\frac{N(K+1)^{2}}{2\left(1+K^{2}\right)} .
$$

Similarly, we obtain the same maximum PAR on the second transmit antenna as in (11). Therefore, to minimize the maximum PAR, DEC and S-DEC should each be implemented on half of the sub-carriers (we refer to this opportunistic hybrid DEC/S-DEC-OFDM scheme by S-DEC-OFDM henceforth). Note that when $m=N$, DEC will be transmitted on all the sub-carriers (we call it DEC-OFDM), which has the same structure as STBC-OFDM. Setting $m=N$ in (9), we obtain that PAR $_{\max }$ of DEC-OFDM is $\frac{2 N K^{2}}{1+K^{2}}$, which is much larger than the $\mathrm{PAR}_{\max }$ of our proposed scheme in (11). When $m=\frac{N}{4}$ or $m=\frac{3 N}{4}$, i.e. S-DEC is implemented on $3 / 4$ or $1 / 4$ of the sub-carriers, $\mathrm{PAR}_{\max }=\frac{N(1+3 K)^{2}}{8\left(K^{2}+1\right)}$, which is smaller than $\mathrm{PAR}_{\max }$ of DEC-OFDM but still larger than $\mathrm{PAR}_{\max }$ of S-DEC-OFDM. Usually, it is the complementary cumulative distribution function (CCDF) which is used as a performance metric for PAR problem. However, the exact closed-form expression of CCDF of PAR defined in (7) is intractable since the elements in vector c are correlated. Therefore, in this paper, we use maximum PAR as a performance metric. The simulation results in Section $\mathrm{V}$ will show that the PAR differences to achieve the same CCDF value are similar to the difference of the maximum PARs in $\mathrm{dB}$ scale.

\section{ICI REDUCTION}

A well-known problem of OFDM is its sensitivity to frequency offset between the transmitter and receiver local carrier frequencies. This frequency offset introduces ICI which affects the error performance. Hence, it is important to design the STBC-OFDM symbol to be robust against this frequency offset. In the previous section, we proposed an opportunistic STBC-OFDM scheme and proved that the lowest PAR is obtained by transmitting each of DEC/S-DEC on half of the sub-carriers. In this section, we will investigate on which subcarriers the DEC and S-DEC schemes should be implemented to reduce ICI introduced by residual carrier frequency offset. Let $\left\{h_{l}: l=0, . ., L-1\right\}$ and $\left\{g_{l}: l=0, . ., L-1\right\}$ denote two independent and identically distributed channels corresponding to the first and the second transmit antennas, respectively. The channel tap gains are assumed to be uncorrelated and constant over two adjacent OFDM symbols. The signal transmitted on the $n$-th sub-carrier in the first time slot on the first antenna is $s_{1, n}$ as given in (3). Then, the signal transmitted on the $n$-th sub-carrier in the first time slot on the 
second antenna is $\bar{s}_{1, n}$ given by

$$
\bar{s}_{1, n}=\left\{\begin{array}{l}
-\frac{b_{1, n}^{*}}{K}, n \in J_{a} \\
-a_{0, n}^{*}, n \in J_{b} .
\end{array}\right.
$$

Thus, the low-pass-equivalent $i$-th received sample at the first time slot after the cyclic prefix removal is [13]

$$
\begin{aligned}
y_{i}= & \frac{1}{N} e^{\frac{j 2 \pi i v}{N}} \sum_{l=0}^{L-1} h_{l} \sum_{n=0}^{N-1} \tilde{s}_{1, n} e^{\frac{j 2 \pi(i-l) n}{N}} \\
& +\frac{1}{N} e^{\frac{j 2 \pi i v}{N}} \sum_{l=0}^{L-1} g_{l} \sum_{n=0}^{N-1} \bar{s}_{1, n} e^{\frac{j 2 \pi(i-l) n}{N}}+w_{i},
\end{aligned}
$$

where $v$ is a frequency offset (normalized by the subcarrier spacing) introduced by the oscillators' inaccuracies and Doppler shift of the mobile wireless channel, and $\left\{w_{i}\right\}$ are independent, circularly-symmetric, zero-mean complex Gaussian noise samples. After FFT operation, the received $n$-th sub-carrier symbol is given by

$$
\begin{aligned}
Y_{n} & =I_{0} \tilde{s}_{1, n} H_{n}+\underbrace{\sum_{m=0, m \neq n}^{N-1} I_{m-n} \tilde{s}_{1, m} H_{m}}_{\mathcal{I}_{1, n}} \\
& +I_{0} \bar{s}_{1, n} G_{n}+\underbrace{\sum_{m=0, m \neq n}^{N-1} I_{m-n} \bar{s}_{1, m} G_{m}}_{\mathcal{I}_{2, n}}+W_{n} \\
& \equiv I_{0} \tilde{s}_{1, n} H_{n}+\mathcal{I}_{1, n}+I_{0} \bar{s}_{1, n} G_{n}+\mathcal{I}_{2, n}
\end{aligned}
$$

where $H_{n}$ and $G_{n}$ are the $n$-th sub-carrier channel gains from the first and second transmit antennas, respectively, $\left\{W_{n}\right\}$ are the frequency-domain Gaussian noise samples corresponding to the time-domain noise samples $\left\{w_{i}\right\}$, and

$$
I_{m-n}=\frac{1}{N} \sum_{k=1}^{N-1} e^{\frac{j 2 \pi(v+m-n) k}{N}} .
$$

Since all the signals are independent and equally likely QPSK symbols, $E\left\{\tilde{s}_{1, m} \bar{s}_{1, n}\right\}=0$ and $E\left\{\mathcal{I}_{1, n} \mathcal{I}_{2, n}\right\}=0$. Then, the variance of the ICI term is

$$
\begin{aligned}
& E\left\{\mathcal{I}_{1, n}+\mathcal{I}_{2, n}\right\}^{2}=E\left\{\left(\mathcal{I}_{1, n}\right)^{2}\right\}+E\left\{\left(\mathcal{I}_{2, n}\right)^{2}\right\} \\
& =E\left\{\sum_{j_{1}=0, j_{1} \neq n}^{N-1} \sum_{j_{2}=0, j_{2} \neq n}^{N-1} I_{j_{1}-n} \tilde{s}_{1, j_{1}} H_{j_{1}} I_{j_{2}-n}^{*} \tilde{s}_{1, j_{2}}^{*} H_{j_{2}}^{*}\right\} \\
& +E\left\{\sum_{j_{1}=0, j_{1} \neq n}^{N-1} \sum_{j_{2}=0, j_{2} \neq n}^{N-1} I_{j_{1}-n} \bar{s}_{1, j_{1}} G_{j_{1}} I_{j_{2}-n}^{*} \bar{s}_{1, j_{2}}^{*} G_{j_{2}}^{*}\right\} .
\end{aligned}
$$

Since

$$
E\left\{\tilde{s}_{1, m} \tilde{s}_{1, n}^{*}\right\}=\left\{\begin{array}{cc}
1 & m=n \in J_{a} \\
\frac{1}{K^{2}} & m=n \in J_{b} \\
0 & m \neq n .
\end{array}\right.
$$

and

$$
E\left\{\bar{s}_{1, m} \bar{s}_{1, n}^{*}\right\}=\left\{\begin{array}{cc}
\frac{1}{K^{2}} & m=n \in J_{a} \\
1 & m=n \in J_{b} \\
0 & m \neq n .
\end{array},\right.
$$

the ICI variance in (16) can be expressed as follows

$$
\begin{aligned}
& E\left\{\left(\mathcal{I}_{1, n}\right)^{2}\right\}+E\left\{\left(\mathcal{I}_{2, n}\right)^{2}\right\} \\
& =\sum_{\substack{m \in J_{a} \\
m=0, m \neq n}}^{N-1}\left\{\left|I_{m-n}\right|^{2} E\left\{\left|H_{m}\right|^{2}\right\}+\frac{\left|I_{m-n}\right|^{2} E\left\{\left|G_{m}\right|^{2}\right\}}{K^{2}}\right\} \\
& +\sum_{\substack{m \in J_{b} \\
m=0, m \neq n}}^{N-1}\left\{\frac{\left|I_{m-n}\right|^{2} E\left\{\left|H_{m}\right|^{2}\right\}}{K^{2}}+\left|I_{m-n}\right|^{2} E\left\{\left|G_{m}\right|^{2}\right\}\right\} \\
& =\sum_{m=0, m \neq n}^{N-1}\left\{\left(1+\frac{1}{K^{2}}\right)\left|I_{m-n}\right|^{2} E\left\{\left|H_{m}\right|^{2}\right\}\right\} .
\end{aligned}
$$

In (18), we have used the relation $E\left\{\left|H_{m}\right|^{2}\right\}=E\left\{\left|G_{m}\right|^{2}\right\}$. From (18), it can be seen that the exact elements of $J_{a}$ and $J_{b}$ do not affect the interference as long as $\left|J_{a}\right|=\left|J_{b}\right|=\frac{N}{2}$. For the second time slot, we get the same result.

\section{Optimization of $K$ OVER Coding Gain And PAR}

Let $X_{a, b, 1}$ and $X_{a, b, 2}$ denote two codewords of DEC with the structure defined in (1) and $G=X_{a, b, 1}-X_{a, b, 2}$ denote the codeword difference matrix. Denote the nonzero eigenvalues of $G G^{H}$ by $\lambda_{1}, \lambda_{2}, \ldots, \lambda_{r}$. Then, the coding gain of Layer A, denoted by $\mathrm{CG}_{\mathrm{A}}$, is defined as

$$
\mathrm{CG}_{\mathrm{A}}=\min _{a_{1} \neq a_{2}} \prod_{i=1}^{r} \lambda_{i}
$$

and the coding gain of Layer $\mathrm{B}$, denoted as $\mathrm{CG}_{\mathrm{B}}$, is defined as

$$
\mathrm{CG}_{\mathrm{B}}=\min _{b_{1} \neq b_{2}} \prod_{i=1}^{r} \lambda_{i}
$$

where $a_{1}$ and $a_{2}$ denote the symbol vectors consisting of all the symbols from Layer $\mathrm{A}$ in codeword $X_{a, b, 1}$ and $X_{a, b, 2}$, respectively. Similarly, $b_{1}$ and $b_{2}$ denote the symbol vectors consisting of all the symbols from Layer B in codeword $X_{a, b, 1}$ and $X_{a, b, 2}$, respectively. A larger power scaling factor $K$ increases the coding gain of Layer A and decreases the coding gain of Layer B as shown in [4]. Also, $K$ will change the maximum PAR given in (11). Therefore, we derive an optimum $K$ according to the design criterion of maximizing the ratio between available coding gain on each sub-carrier and the maximum PAR. Different from the classical STBCOFDM, there is a trade-off between the coding gain of Layer $A$ and that of Layer B for DEC-OFDM. Therefore, our design criterion must take into consideration the coding gain of both layers. From [4] and [1], we know that the pairwise error probability (PEP) of Layer A and Layer B can be, respectively, upper bounded as follows

$$
P_{A}\left(X_{a, b, 1} \rightarrow X_{a, b, 2}\right) \leq\left(\mathrm{CG}_{\mathrm{A}}\right)^{-M_{r}}\left(E_{s} / N_{0}\right)^{-r_{A} M_{r}},
$$

and

$$
P_{B}\left(X_{a, b, 1} \rightarrow X_{a, b, 2}\right) \leq\left(\mathrm{CG}_{\mathrm{B}}\right)^{-M_{r}}\left(E_{s} / N_{0}\right)^{-r_{B} M_{r}},
$$

where $M_{r}$ is the number of receive antennas, $r_{A} M_{r}$ and $r_{B} M_{r}$ are the diversity orders of Layers $\mathrm{A}$ and $\mathrm{B}$, respectively. We use the geometric average of $P_{A}$ and $P_{B}$ as our single 


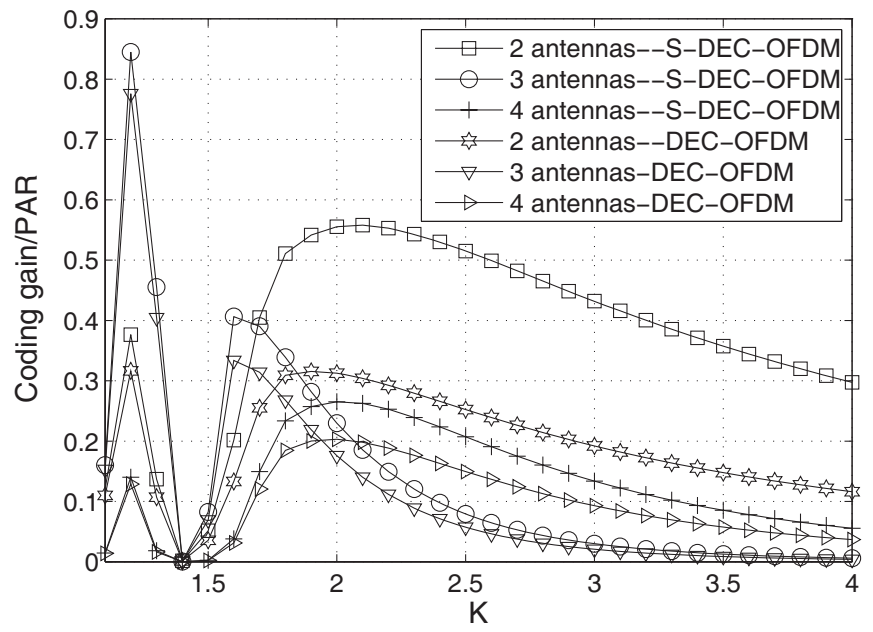

Fig. 2. Joint optimization of coding gain and PAR.

performance measure for the error performance of both layers. Note that the arithmetic average of $P_{A}$ and $P_{B}$ is not an appropriate criterion since the performance of the layer with larger error probability will dominate the criterion. Therefore, we get

$$
\sqrt{P_{A} P_{B}} \leq\left(\sqrt{\mathrm{CG}_{\mathrm{A}} \mathrm{CG}_{\mathrm{B}}}\left(\frac{E_{s}}{N_{0}}\right)^{-\frac{r_{A}+r_{B}}{2}}\right)^{-M_{r}} .
$$

Since $K$ affects the coding gains only, minimizing the geometric average of $P_{A}$ and $P_{B}$ is equivalent to maximizing the geometric average of the coding gains of Layer $\mathrm{A}$ and B. Note that the same coding gain is achieved for DEC and S-DEC. On the other hand, a larger PAR $\mathrm{Pax}_{\max }$ causes a larger performance loss/power loss. Then, our design criterion becomes

$$
K_{\mathrm{opt}}=\underset{K>1}{\operatorname{argmax}}\left\{\frac{\sqrt{\mathrm{CG}_{\mathrm{A}} \mathrm{CG}_{\mathrm{B}}}}{\frac{\mathrm{PAR}_{\max }}{N}}\right\},
$$

where $\frac{\mathrm{PAR}_{\max }}{N}$ is the normalized maximum PAR in (11). Fig. 2 shows this design metric as a function of $K$ for the QPSK constellation. We observe that the optimum $K$ is around 2 for 2 transmit antennas. In Fig. 2, the corresponding design metric for DEC-OFDM is also given where $\frac{\mathrm{PAR}_{\max }}{N}=\frac{2 K^{2}}{1+K^{2}}$. The optimum $K$ for DEC-OFDM is also around 2.

\section{Simulation Results}

In this section, we compare the performance of the following five transmission schemes:

- DEC-OFDM: $J_{a}=N$.

- Block S-DEC-OFDM: $J_{a}=\left\{0,1, \ldots, \frac{N}{2}-1\right\}$ and $J_{b}=$ $\left\{\frac{N}{2}, \ldots, N-1\right\}$.

- Interleaved S-DEC-OFDM: $J_{a}=\{0,2, \ldots, N-2\}$ and $J_{b}=\{1,3, \ldots, N-1\}$.

- 1/4 block S-DEC-OFDM: $J_{a}=\left\{0,1, \ldots, \frac{N}{4}-1\right\}$ and $J_{b}=\left\{\frac{N}{4}, \ldots, N-1\right\}$.

- 3/4 block S-DEC-OFDM: $J_{a}=\left\{0,1, \ldots, \frac{3 N}{4}-1\right\}$ and $J_{b}=\left\{\frac{3 N}{4}, \ldots, N-1\right\}$.

In this simulation, $N=64$ and the OFDM symbol is oversampled by a factor of 8 [14].

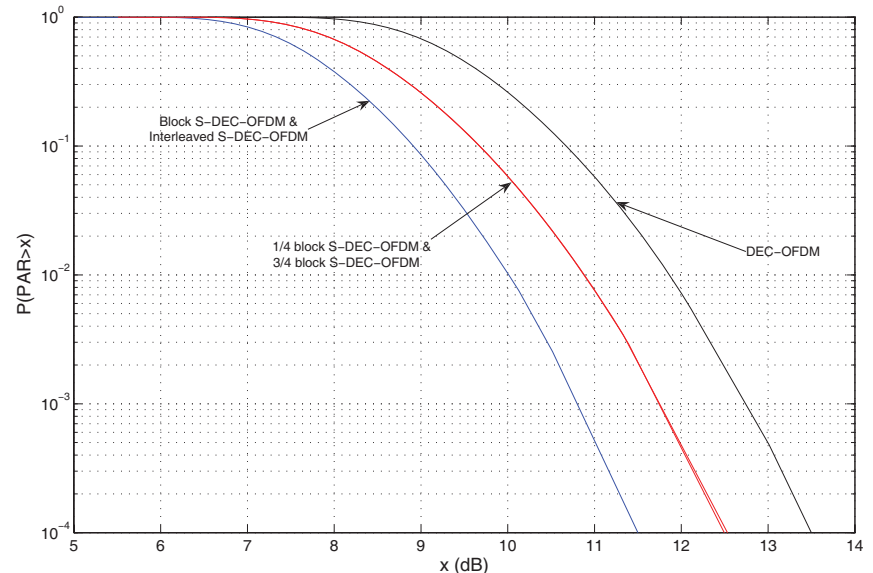

Fig. 3. CCDF comparison with different switch schemes.

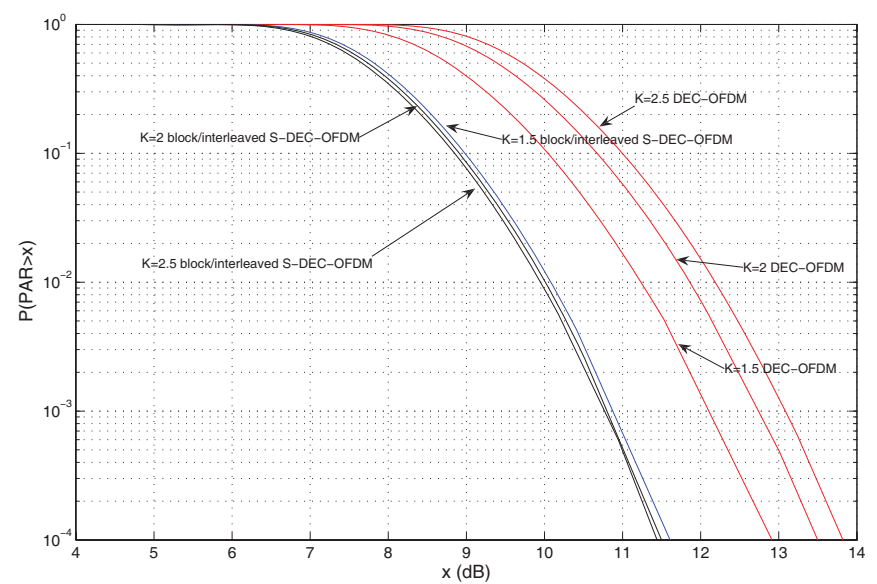

Fig. 4. CCDF comparison with different K.

\section{A. CCDF Comparison}

The CCDF of the PAR is calculated over 1,000,000 DECOFDM block realizations. Fig. 3 compares the CCDF of the four schemes at $K=2$, where it can be seen that the PAR of the block S-DEC-OFDM and interleaved S-DEC-OFDM are identical and both are about $2 \mathrm{~dB}$ lower than that of DECOFDM. The PAR of the $1 / 4$ block S-DEC-OFDM and $3 / 4$ block S-DEC-OFDM are worse than block S-DEC-OFDM and better than that of DEC-OFDM, which is consistent with our derived $\mathrm{PAR}_{\max }$ relation for these five schemes given in Section II. From (11), we know that $\mathrm{PAR}_{\max }$ decreases with increasing $K$. From Fig. 4, it can also be seen that a larger $K$ yields a smaller PAR of S-DEC-OFDM. However, for DECOFDM, the larger the value of $K$ is, the larger the PAR is.

\section{B. BER Performance with ICI Reduction}

In this subsection, the channels are modeled as independent 3-tap Rayleigh fading channels with an exponential power delay profile. In our simulation, we use $K=2$ and a frequency offset (normalized by the sub-carrier spacing) $v=0.01$. At the receiver, the maximum likelihood interference cancellation (MLIC) algorithm proposed in [5] is used to decode the information symbols. Fig. 5 shows that DEC-OFDM, block S-DEC-OFDM and interleaved S-DEC-OFDM have the same BER performance, which corroborates our ICI analysis in 


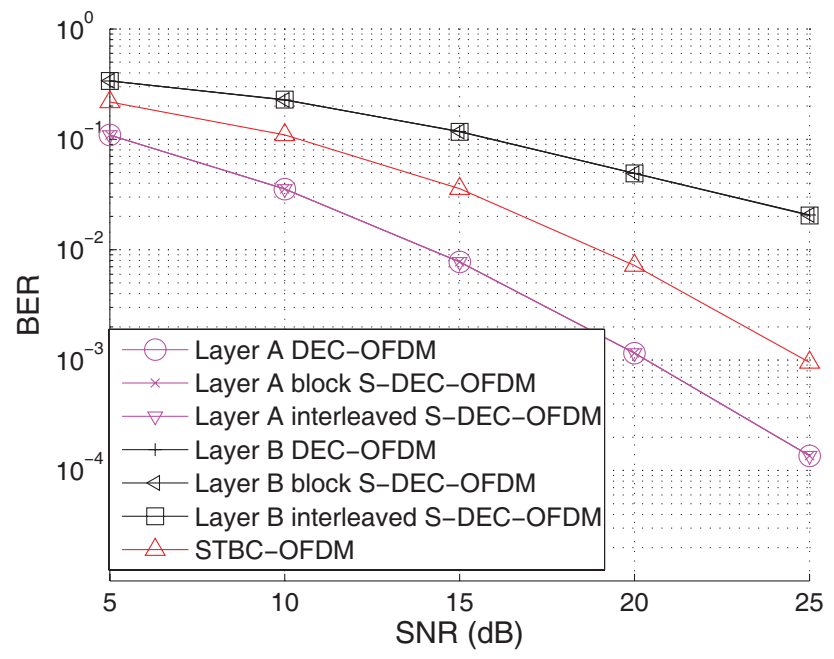

Fig. 5. BER performance comparison at $K=2$.

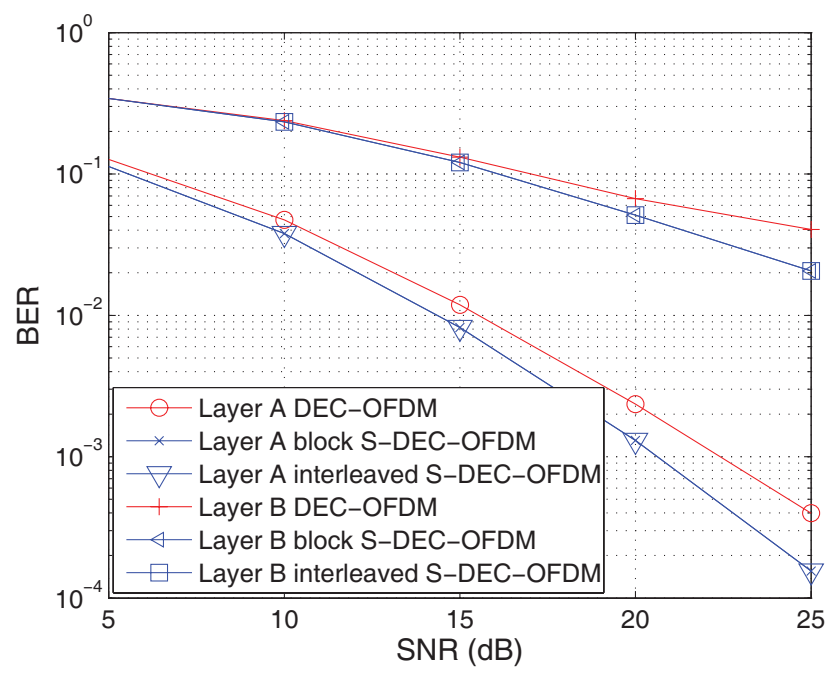

Fig. 6. BER performance comparison at $K=2$ with the PAR clipping threshold of $5 \mathrm{~dB}$.

Section III. The performance of the classical STBC-OFDM with the Alamouti STBC [15] is also given as the benchmark. Similar to the conclusions in [4], the BER of single-layer Alamouti is "sandwiched" between the BERs of the 2 layers of S-DEC-OFDM and DEC-OFDM.

\section{BER Performance with Clipping}

In this subsection, we use clipping and FFT-based filtering prior to transmission to evaluate the considered schemes in practical systems. Clipping and filtering are simple and effective practical methods for addressing PAR issues ([16] [17] [18]). After we obtained the signals $s_{1, p}, s_{2, p}$ as in (4), the signal samples larger than a certain threshold were clipped, i.e., the clipped signals in the time domain are given by

$$
\hat{c}_{1, p}=\left\{\begin{array}{cl}
s_{1, p} & \left|s_{1, p}\right| \leq \sqrt{\frac{1+\frac{1}{K^{2}}}{2 N M^{2}} 10^{\frac{\Gamma}{10}}} \\
\sqrt{\frac{1+\frac{1}{K^{2}}}{2 N M^{2}} 10^{\frac{\Gamma}{10}}} e^{j \phi} & \left|s_{1, p}\right|>\sqrt{\frac{1+\frac{1}{K^{2}}}{2 N M^{2}} 10^{\frac{\Gamma}{10}}}
\end{array},\right.
$$

where $\phi$ is the phase of $s_{1, p}$ and $\Gamma$ is the PAR clipping threshold. After clipping, to keep the same bandwidth, an $N M$ point FFT is implemented on $\hat{c}_{1, p}$ and only the first $N$ point outputs of the FFT represent the actual transmission signals in the frequency domain. We assume that the amplifier linearity range is slightly larger than the PAR clipping threshold to account for the peak regrowth due to filtering. It can be seen from Fig. 6 that at the PAR clipping threshold of $5 \mathrm{~dB}$, by simply switching the signals on two mini-slots, our proposed block S-DEC-OFDM and interleaved S-DEC-OFDM achieve about $2 \mathrm{~dB}$ SNR gain at $\mathrm{BER}=10^{-3}$ over DEC-OFDM of Layer A.

\section{EXTENSION TO 3 AND 4 TRANSMit ANTENNAS}

In this section, opportunistic STBC-OFDM designs for 3 and 4 transmit antennas are presented. Due to the space limitations, the corresponding detailed performance analysis is not given. The DEC code for 3 transmit antennas [4] is given by

$$
X_{a, b, c}=\left(\begin{array}{cccc}
a(0) & -a^{*}(1) & -a^{*}(2) & \frac{c^{*}(0)}{K} \\
a(1) & a^{*}(0) & \frac{b^{*}(0)}{K} & -a^{*}(2) \\
a(2) & \frac{b^{*}(0)}{K} & a^{*}(0) & a^{*}(1)
\end{array}\right) .
$$

Let $a_{0, n}, a_{1, n}, a_{2, n}, b_{0, n}$ and $c_{0, n}$ denote the signal $a(0)$, $a(1), a(2), b(0)$ and $c(0)$, respectively, associated with the $n$-th $(n=0,1, \ldots, N-1)$ sub-carrier. Let $\mathbf{x}_{1, \mathbf{n}}, \mathbf{x}_{\mathbf{2}, \mathbf{n}}$, $\mathbf{x}_{\mathbf{3}, \mathbf{n}}$ and $\mathbf{x}_{\mathbf{4}, \mathbf{n}}$ denote the corresponding signal vectors on the four time slots respectively, associated with the three transmit antennas, i.e. $\mathbf{x}_{\mathbf{1}, \mathbf{n}}=\left[a_{0, n}, a_{1, n}, a_{2, n}\right]^{T}, \mathbf{x}_{\mathbf{2}, \mathbf{n}}=$ $\left[-a_{1, n}^{*}, a_{0, n}^{*}, \frac{b_{0, n}^{*}}{K}\right]^{T}, \mathbf{x}_{\mathbf{3}, \mathbf{n}}=\left[-a_{2, n}^{*}, \frac{b_{0, n}^{*}}{K}, a_{0, n}^{*}\right]^{T}$ and $\mathbf{x}_{\mathbf{4}, \mathbf{n}}=$ $\left[\frac{c_{0, n}^{*}}{K},-a_{2, n}^{*}, a_{1, n}^{*}\right]^{T}$. Then, one possible block S-DEC-OFDM is given as

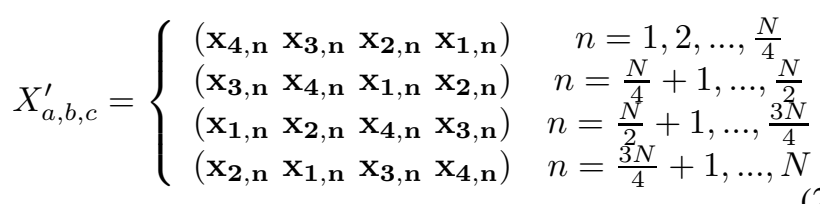

The optimum $K$ of S-DEC-OFDM and DEC-OFDM are almost the same which is around 1.2 as shown in Fig. 2.

Similarly, the DEC code for 4 transmit antennas [4] is given by

$$
X_{a, b}=\left(\begin{array}{cccc}
a(0) & -a^{*}(1) & -a^{*}(2) & \frac{b(1)}{K} \\
a(1) & a^{*}(0) & \frac{b^{*}(0)}{K} & -a^{*}(2) \\
a(2) & \frac{b^{*}(0)}{K} & a^{*}(0) & a^{*}(1) \\
\frac{b(1)}{K} & a(2) & -a(1) & a(0)
\end{array}\right) .
$$

Here, we define $a_{0, n}, a_{1, n}, a_{2, n}, b_{0, n}$ and $b_{1, n}$ as the signal $a(0), a(1), a(2), b(0)$ and $b(1)$, respectively, associated with the $n$-th $(n=0,1, \ldots, N-1)$ sub-carrier. Let $\mathbf{x}_{1, n}, \mathbf{x}_{2, n}, \quad \mathbf{x}_{3, n}$ and $\mathbf{x}_{4, \mathbf{n}}$ denote the corresponding signal vectors on the four time slots respectively, associated with the four transmit antennas, i.e. $\mathbf{x}_{\mathbf{1}, \mathbf{n}}=$ $\left[a_{0, n}, a_{1, n}, a_{2, n}, \frac{b_{1, n}}{K}\right]^{T}, \mathbf{x}_{\mathbf{2}, \mathbf{n}}=\left[-a_{1, n}^{*}, a_{0, n}^{*}, \frac{b_{0, n}^{*}}{K}, a_{2, n}\right]^{T}$, $\mathbf{x}_{\mathbf{3}, \mathbf{n}}=\left[-a_{2, n}^{*}, \frac{b_{0, n}^{*}}{K}, a_{0, n}^{*},-a_{1, n}\right]^{T}$ and $\mathbf{x}_{\mathbf{4}, \mathbf{n}}=$ $\left[\frac{b_{1, n}}{K},-a_{2, n}^{*}, a_{1, n}^{*}, a_{0, n}\right]^{T}$. Then, one possible block S- 
DEC-OFDM is given as

$$
X_{a, b}^{\prime}=\left\{\begin{array}{cc}
\left(\mathbf{x}_{4, \mathbf{n}} \mathbf{x}_{\mathbf{3}, \mathbf{n}} \mathbf{x}_{\mathbf{2}, \mathbf{n}} \mathbf{x}_{\mathbf{1}, \mathbf{n}}\right) & n=1,2, \ldots, \frac{N}{4} \\
\left(\mathbf{x}_{\mathbf{3}, \mathbf{n}} \mathbf{x}_{\mathbf{4}, \mathbf{n}} \mathbf{x}_{\mathbf{1}, \mathbf{n}} \mathbf{x}_{\mathbf{2}, \mathbf{n}}\right) & n=\frac{N}{4}+1, \ldots, \frac{N}{2} \\
\left(\mathbf{x}_{\mathbf{1}, \mathbf{n}} \mathbf{x}_{\mathbf{2}, \mathbf{n}} \mathbf{x}_{\mathbf{4}, \mathbf{n}} \mathbf{x}_{\mathbf{3}, \mathbf{n}}\right) & n=\frac{N}{2}+1, \ldots, \frac{3 N}{4} \\
\left(\mathbf{x}_{\mathbf{2}, \mathbf{n}} \mathbf{x}_{\mathbf{1}, \mathbf{n}} \mathbf{x}_{\mathbf{3}, \mathbf{n}} \mathbf{x}_{\mathbf{4}, \mathbf{n}}\right) & n=\frac{3 N}{4}+1, \ldots, N
\end{array}\right.
$$

It can be seen from Fig. 2 that the optimum $K$ is around 2, which is the same for both S-DEC-OFDM and DEC-OFDM.

Please note that the S-DEC-OFDM structure for 3 and 4 antennas are not unique as long as it has the same average power on different OFDM symbols.

\section{CONCLUSIONS}

In this paper, we propose an opportunistic STBC-OFDM scheme for 2 transmit antennas with reduced PAR in the presence of carrier frequency offset. Our theoretical analysis shows that the lowest PAR is achieved when DEC and S-DEC are each implemented on half of the sub-carriers. ICI analysis shows that, in the presence of carrier frequency offset, subcarrier indices on which DEC or S-DEC is implemented do not affect the BER performance. Furthermore, we optimize the scheme by selecting the optimum power scaling factor $K$ to achieve the best tradeoff between coding gain and PAR. For QPSK modulation, at the optimum power scaling factor $(K=2)$, the PAR of S-DEC-OFDM is $2 \mathrm{~dB}$ less than that of DEC-OFDM. When clipping and filtering are implemented for PAR reduction at the transmitter, S-DEC-OFDM can achieve about $2 \mathrm{~dB}$ SNR gain over DEC-OFDM at the PAR clipping threshold of $5 \mathrm{~dB}$. Extension to 3 and 4 transmit antennas are also presented.

\section{REFERENCES}

[1] V. Tarokh, N.Seshadri, and A. Calderbank, "Space-time codes for high data rate wireless communications: performance criterion and code construction," IEEE Trans. Inform. Theory, vol. 44, no. 2, pp. 744-765, Mar. 1998.

[2] S. Diggavi, N. Al-Dhahir, A. Stamoulis, and A. Calderbank, "Great expectations : The value of spatial diversity in wireless networks," in Proc. IEEE (Special Issue on Gigabit Wireless), vol. 92, Feb. 2004, pp. 219-270.
[3] S. Diggavi, N. Al-Dhahir, and A. Calderbank, "Diversity-embedded space-time codes," in Proc. IEEE Globecom, vol. 4, Dec. 2003, pp. 1909-1914.

[4] S. Das and N. Al-Dhahir, "New diversity-embedding space-time codes constructions," in Proc. SPAWC, July 2006.

[5] S. Das, N. Al-Dhahir, S. Diggavi, and A. Calderbank, "Opportunistic space-time block codes," in Proc. IEEE VTC conf., vol. 3, Fall 2005, pp. 2025-2029.

[6] N. Al-Dhahir, "Overview and comparison of equalization schemes for space-time-coded signals with application to EDGE," IEEE Trans. Signal Processing (special issue), vol. 50, pp. 2477-2488, Oct. 2002.

[7] A. D. S. Jayalath and C. Tellambura, "Use of data permutation to reduce the peak-to-average power ratio of an OFDM signal," Wireless Commun. Mobile Comput. J., vol. 2, pp. 187-203, Mar. 2002.

[8] R. W. Bauml, R. F. H. Fischer, and J. B. Huber, "Reducing the peaktoaverage power ratio of multicarrier modulation by selected mapping," Electron. Lett., vol. 32, pp. 2056-2057, Oct. 1996.

[9] J. Armstrong, "Analysis of new and existing methods of reducing intercarrier interference due to carrier frequency offset in OFDM," IEEE Trans. Commun., vol. 47, no. 3, pp. 365-369, 1999.

[10] A. Seyedi and G. J. Saulnier, "General ICI self-cancellation scheme for OFDM systems," IEEE Trans. Veh. Technol., vol. 54, pp. 198-210, Jan. 2005.

[11] H. Minn, N. Al-Dhahir, and Y. Li, "Optimal training signals for MIMO OFDM channel estimation in the presence of frequency offsets and phase noise," IEEE Trans. Commun., vol. 54, pp. 1754-1759, Oct. 2006.

[12] J. G. Proakis and D. G. Manolakis, Digital Signal Processing: Principle, Algorithms, and Applications, 3rd ed. Prentice Hall, 1995.

[13] X. Fu and H. Minn, "Modified data-pilot-multiplexed schemes for OFDM systems," IEEE Trans. Wireless Commun., vol. 6, pp. 730-737, Feb. 2007.

[14] C. Tellambura, "Computation of the continuous-time PAR of an OFDM signal with BPSK subcarriers," IEEE Commun. Lett., vol. 5, pp. 185187, May 2001

[15] S. M. Alamouti, "A simple transmit diversity technique for wireless communications," IEEE J. Select. Areas Commun., vol. 16, pp. 14511458 , Oct. 1998

[16] X. Li and L. J. Cimini, "Effects of clipping and filtering on the perfromance of OFDM," IEEE Commun. Lett., vol. 2, no. 5, pp. 131133, May 1998

[17] J. Armstrong, "Peak-to-average power reduction for OFDM by repeated clipping and frequency domain filtering," Electron. Lett., vol. 38, pp. 246-247, Feb. 2002.

[18] L. Wang and C. Tellambura, "A simplified clipping and filtering technique for PAR reduction in OFDM systems," IEEE Signal Process. Lett., vol. 12, pp. 453-456, June 2005. 